MULTIPLE SCLEROSIS

\section{Microstructural pathology imaged in MS}

The variability of axon and dendrite orientations - termed neurite orientation dispersion - is reduced in the spinal cords of patients with multiple sclerosis (MS), according to new research. This finding could provide a new biomarker for MS prognosis and therapeutic efficacy.

The clinical manifestation, disease course, and severity of MS are highly variable, making the search for new biomarkers crucial. Although inflammation is a central hallmark in MS, other pathological mechanisms exist. "Recent work has demonstrated that the complexity of axon and dendrite architecture carries a signature of MS pathology. We therefore decided to test whether neurite orientation dispersion is a useful biomarker in MS, as to date, conventional MRI readouts provide limited information about clinical prognosis", explains Gabriele De Luca, co-senior author of the study with Gandini Wheeler-Kingshott.

The researchers used a stateof-the-art, clinically viable, MRI technique, called neurite orientation dispersion and density imaging (NODDI), and histological investigations, to study neurite architecture, in

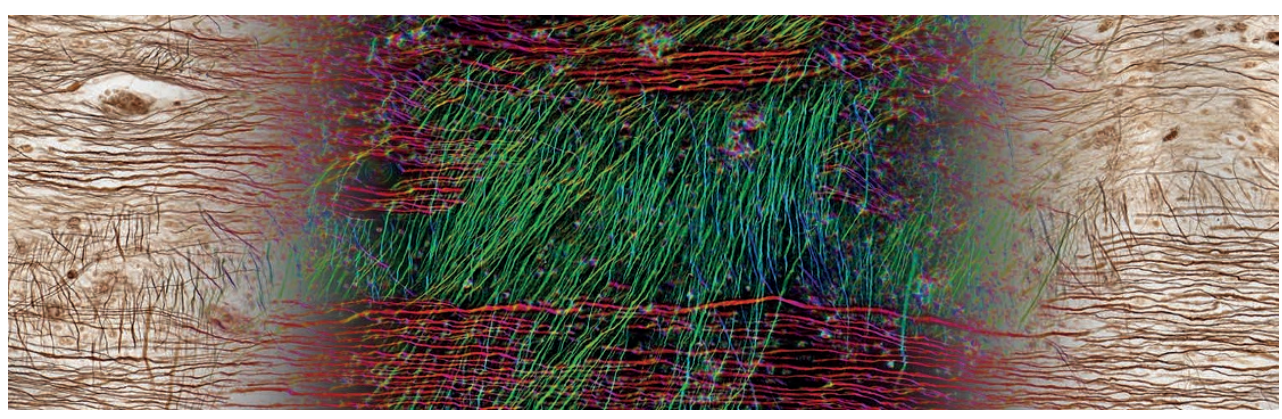

A sagittal spinal cord section (top of spinal cord to left) stained with the Palmgren's silver method, which labels axons dark brown. In the centre, axons have been coloured according to their orientation, calculated by Structure Tensor Analysis (STA). The local variability of these orientations at the MRI voxel scale represents orientation dispersion. Image courtesy of F. Grussu. four human post-mortem spinal cord specimens - two from patients with progressive MS and two from healthy controls. "Spinal cord tissue was analysed in a completely novel way, by looking at key neuropathological elements - astrocyte, microglia, myelin and neurofilament density — from a sagittal view, to get a better idea of the architecture of nerve cells", explains De Luca. Analyses showed reduced orientation variability of neurites within focal areas of demyelination in MS, with NODDI and histology, indicating reduced branching or morphological alterations of individual axons in white matter, and reduced complexity of dendritic arborisations in grey matter, compared to non-lesional tissue.

"NODDI-derived dispersion was sensitive and specific to its histological counterpart, which sets the stage for understanding clinically relevant nerve fibre-related changes in MS that could only be detected previously post-mortem" De Luca concludes. The research team now intends to apply these imaging techniques to people with varying degrees of MS, to determine if they have a clinical correlate.

Mitesh Patel

ORIGINAL ARTICLE Grussu, F. et al. Neurite dispersion: a new marker of multiple sclerosis spinal cord pathology?. Annals of Clinical and Translational Neurology. http://onlinelibrary.wiley. com/doi/10.1002/acn3.445/full (2017) 